\title{
Parallel Multi-party Computation from Linear Multi-secret Sharing Schemes ${ }^{\star}$
}

\author{
Zhifang Zhang ${ }^{1}$, Mulan $\mathrm{Liu}^{1, \star \star}$, and Liangliang Xiao ${ }^{2}$ \\ ${ }^{1}$ Academy of Mathematics and Systems Science, \\ Key Laboratory of Mathematics Mechanization, \\ Chinese Academy of Sciences, \\ Beijing, 100080, China \\ $\{z f z$, mlliu\}@amss.ac.cn \\ ${ }^{2}$ Institute of Software, Chinese Academy of Sciences, \\ Beijing, 100080, China \\ xllemail2004@yahoo.com.cn
}

\begin{abstract}
As an extension of multi-party computation (MPC), we propose the concept of secure parallel multi-party computation which is to securely compute multi-functions against an adversary with multistructures. Precisely, there are $m$ functions $f_{1}, \ldots, f_{m}$ and $m$ adversary structures $\mathcal{A}_{1}, \ldots, \mathcal{A}_{m}$, where $f_{i}$ is required to be securely computed against an $\mathcal{A}_{i}$-adversary. We give a general construction to build a parallel multi-party computation protocol from any linear multi-secret sharing scheme (LMSSS), provided that the access structures of the LMSSS allow MPC at all. When computing complicated functions, our protocol has more advantage in communication complexity than the "direct sum" method which actually executes a MPC protocol for each function. The paper also provides an efficient and generic construction to obtain from any LMSSS a multiplicative LMSSS for the same multi-access structure.
\end{abstract}

\section{Introduction}

The secure multi-party computation (MPC) protocol is used for $n$ players to jointly compute an agreed function of their private inputs in a secure way, where security means guaranteeing the correctness of the output and the privacy of the players' inputs, even when some players cheat. It is fundamental in cryptography and distributed computation, because a solution of MPC problem implies in principle a solution to any cryptographic protocol problem, such as the voting problem, blind signature, and so on. After it was proposed by Yao 11 for twoparty case and Goldreich, Micali, Wigderson [6] for multi-party case, it has become an active and developing field of information security.

In the MPC problem, it is common to model cheating by considering an adversary who may corrupt some subset of the players. The collection of all subsets

\footnotetext{
* Supported by the National Natural Science Foundation of China (No. 90304012, 90204016), 973 project (No. 2004CB318000).

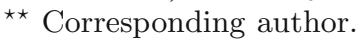


that an adversary may corrupt is called the adversary structure, denoted by $\mathcal{A}$, and this adversary is called an $\mathcal{A}$-adversary. So the MPC problem is to securely compute a function with respect to an adversary structure. But in practice it is sometimes needed to simultaneously compute several different functions with respect to different adversary structures, respectively. For example, in the voting problem $n=2 t+1(t>1)$ voters are to select a chairman and several fellows for a committee at the same time from $m$ candidates. Because the position of the chairman is more important than that of fellows, the voting for the chairman is required to be secure against a $(t, n)$ threshold adversary, while the voting for the fellows is required to be secure against a $(2, n)$ threshold adversary. Hence it makes us to propose parallel multi-party computation or extend MPC to parallel MPC. Precisely, in the problem of parallel multi-party computation, there are $m$ functions $f_{1}, \ldots, f_{m}$ and $m$ adversary structures $\mathcal{A}_{1}, \ldots, \mathcal{A}_{m}$, where $f_{i}$ is required to be securely computed against an $\mathcal{A}_{i}$-adversary.

Obviously, secure parallel multi-party computation can be realized by designing for each function a MPC protocol with respect to the corresponding adversary structure, and then running all the protocols in a composite way. We call this the "direct sum" method. In this paper, we propose another way to realize parallel multi-party computation. It is well known that secret sharing schemes are elementary tool for studying MPC. Cramer, Damgard, Maurer 3] gave a generic and efficient construction to build a MPC protocol from any linear secret sharing scheme (LSSS). As an extension of secret sharing schemes, Blundo, De Santis, Di Crescenzo [2 proposed the general concept of multi-secret sharing schemes which is to share multi-secrets with respect to multi-access structures, and Ding, Laihonen, Renvall. [4 studied linear multisecret sharing schemes. Based on Xiao and Liu's work [10] about linear multisecret sharing schemes (LMSSS) and the construction in [3, we give a generic and efficient construction to build a parallel multi-party computation protocol from any LMSSS, provided that the access structures of the LMSSS allow MPC at all 7. We only deal with adaptive, passive adversaries in the information theoretic model. When computing complicated functions, our protocol has more advantage in communication complexity than the "direct sum" method.

The paper is organized as follows: in Section 2 we review some basic concepts, such as LSSS, monotone span programs (MSP) and LMSSS. In Section 3 we give a clear description for the problem of secure parallel multi-party computation, and then obtain a generic protocol for it from any LMSSS. Furthermore we compare our protocol with the "direct sum" method in communication complexity. In the last section, a specific example is displayed in detail to show how our protocol works as well as its advantage.

\section{Preliminaries}

Since secret sharing schemes are our primary tool, first we review some basic concepts and results about them, such as linear secret sharing schemes, 
multi-secret sharing schemes, monotone span programs, and so on. Suppose that $P=\left\{P_{1}, \ldots, P_{n}\right\}$ is the set of participants and $\mathcal{K}$ is a finite field throughout this paper.

\subsection{LSSS vs MSP}

It is well-known that an access structure, denoted by $A S$, is a collection of subsets of $P$ satisfying the monotone ascending property: for any $A^{\prime} \in A S$ and $A \in 2^{P}$ with $A^{\prime} \subset A$, it holds that $A \in A S$; and an adversary structure, denoted by $\mathcal{A}$, is a collection of subsets of $P$ satisfying the monotone descending property: for any $A^{\prime} \in \mathcal{A}$ and $A \in 2^{P}$ with $A \subset A^{\prime}$, it holds that $A \in \mathcal{A}$. In this paper, we consider the complete situation, i.e. $\mathcal{A}=2^{P}-A S$. Because of the monotone property, for any access structure $A S$ it is enough to consider the minimum access structure $A S_{m}$ defined as $A S_{m}=\{A \in A S \mid \forall B \varsubsetneqq A \Rightarrow B \notin A S\}$.

Suppose that $S$ is the secret-domain, $R$ is the set of random inputs, and $S_{i}$ is the share-domain of $P_{i}$ where $1 \leq i \leq n$. A secret sharing scheme with respect to an access structure $A S$ is composed of the distribution function $\Pi$ : $S \times R \rightarrow S_{1} \times \cdots \times S_{n}$ and the reconstruction function: for any $A \in A S$, $R e=\left\{\operatorname{Re}_{A}:\left.\left(S_{1} \times \cdots \times S_{n}\right)\right|_{A} \rightarrow S \mid A \in A S\right\}$, such that the following two requirements are satisfied.

(i) Correctness requirement: for any $A \in A S, s \in S$ and $r \in R$, it holds that $\operatorname{Re}_{A}\left(\left.\Pi(s, r)\right|_{A}\right)=s$, where suppose $A=\left\{P_{i_{1}}, \ldots, P_{i_{|A|}}\right\}$ and $\Pi(s, r)=$ $\left(s_{1}, \ldots, s_{n}\right)$, then $\left.\Pi(s, r)\right|_{A}=\left(s_{i_{1}}, \ldots, s_{i_{|A|}}\right)$.

(ii) Security requirement: for any $B \notin A S$, i.e., $B \in \mathcal{A}=2^{P} \backslash A S$, it holds that $0<H\left(S|\Pi(S, R)|_{B}\right) \leq H(S)$, where $H(\cdot)$ is the entropy function.

In the security requirement, if $H\left(S|\Pi(S, R)|_{B}\right)=H(S)$, we call it a perfect secret sharing scheme which we are interested in. Furthermore, a perfect secret sharing scheme is linear (LSSS for short), if $S, R, S_{i}$ are all linear spaces over $\mathcal{K}$ and the reconstruction function is linear 1 .

Karchmer and Wigderson [8] introduced monotone span programs (MSP) as linear models computing monotone Boolean functions. Usually we denote a MSP by $\mathcal{M}(\mathcal{K}, M, \psi)$, where $M$ is a $d \times l$ matrix over $\mathcal{K}$ and $\psi:\{1, \ldots, d\} \rightarrow\left\{P_{1}, \ldots, P_{n}\right\}$ is a surjective labelling map which actually distributes to each participant some rows of $M$. We call $d$ the size of the MSP. For any subset $A \subseteq P$, there is a corresponding characteristic vector $\overrightarrow{\delta_{A}}=\left(\delta_{1}, \ldots, \delta_{n}\right) \in\{0,1\}^{n}$ where for $1 \leq$ $i \leq n, \delta_{i}=1$ if and only if $P_{i} \in A$. Consider a monotone Boolean function $f:\{0,1\}^{n} \rightarrow\{0,1\}$ which satisfies that for any $A \subseteq P$ and $B \subseteq A, f\left(\overrightarrow{\delta_{B}}\right)=1$ implies $f\left(\overrightarrow{\delta_{A}}\right)=1$. We say that a $\operatorname{MSP} \mathcal{M}(\mathcal{K}, M, \psi)$ computes the monotone Boolean function $f$ with respect to a target vector $\vec{v} \in \mathcal{K}^{l} \backslash\{(0, \ldots, 0)\}$, if it holds that $\vec{v} \in \operatorname{span}\left\{M_{A}\right\}$ if and only if $f\left(\overrightarrow{\delta_{A}}\right)=1$, where $M_{A}$ consists of the rows $i$ of $M$ with $\psi(i) \in A$ and $\vec{v} \in \operatorname{span}\left\{M_{A}\right\}$ means that there exists a vector $\vec{w}$ such that $\vec{v}=\vec{w} M_{A}$. Beimel [1] proved that devising a LSSS with respect to an access structure $A S$ is equivalent to constructing a MSP computing the monotone Boolean function $f_{A S}$ which satisfies $f_{A S}\left(\overrightarrow{\delta_{A}}\right)=1$ if and only if $A \in A S$. 


\subsection{LMSSS vs MSP}

Multi-Secret sharing schemes 2] are to share multi-secrets with respect to multiaccess structures. Precisely, let $A S_{1}, \ldots, A S_{m}$ be $m$ access structures over $P$, $S^{1} \times \cdots \times S^{m}$ be the secret-domain, $S_{1}, \ldots, S_{n}$ be the share-domain and $R$ be the set of random inputs. Without loss of generality, we assume that $S^{1}=\cdots=$ $S^{m}=\mathcal{K}$. A linear multi-secret sharing scheme (LMSSS for short) realizing the multi-access structure $A S_{1}, \cdots, A S_{m}$ is composed of the distribution function

$$
\begin{gathered}
\Pi: \mathcal{K}^{m} \times R \longrightarrow S_{1} \times \cdots \times S_{n} \\
\Pi\left(s^{1}, \cdots, s^{m}, r\right)=\left(\Pi_{1}\left(s^{1}, \cdots, s^{m}, r\right), \cdots, \Pi_{n}\left(s^{1}, \cdots, s^{m}, r\right)\right),
\end{gathered}
$$

and the reconstruction function $\operatorname{Re}=\left\{\operatorname{Re}_{A}^{i}:\left(S_{1} \times \cdots \times S_{n}\right)_{A} \rightarrow \mathcal{K} \mid 1 \leq i \leq\right.$ $\left.m, A \in A S_{i}\right\}$, such that the following three conditions hold:

(i) $S_{1}, \cdots, S_{n}$ and $R$ are finitely dimensional linear spaces over $\mathcal{K}$, i.e., there exist positive integers $d_{k}, 1 \leq k \leq n$, and $l$ such that $S_{k}=\mathcal{K}^{d_{k}}$ and $R=\mathcal{K}^{l}$. Precisely, in the equality (1), we have that $\Pi_{k}\left(s^{1}, \cdots, s^{m}, r\right) \in \mathcal{K}^{d_{k}}$ for $1 \leq k \leq$ $n$. Furthermore, denote

$$
\Pi_{k}\left(s^{1}, \cdots, s^{m}, r\right)=\left(\Pi_{k 1}\left(s^{1}, \cdots, s^{m}, r\right), \cdots, \Pi_{k d_{k}}\left(s^{1}, \cdots, s^{m}, r\right)\right)
$$

where $\Pi_{k j}\left(s^{1}, \cdots, s^{m}, r\right) \in \mathcal{K}$ and $1 \leq j \leq d_{k}$. Usually $d=\sum_{i=1}^{n} d_{i}$ is called the size of the linear multi-secret sharing scheme.

(ii) The reconstruction function is linear. That is, for any set $A \in A S_{i}, 1 \leq$ $i \leq m$, there exists a set of constants $\left\{\alpha_{k j}^{i} \in \mathcal{K} \mid 1 \leq k \leq n, P_{k} \in A, 1 \leq j \leq d_{k}\right\}$ such that for any $s^{1}, \ldots, s^{m} \in \mathcal{K}$ and $r \in R, s^{i}=\operatorname{Re}_{A}^{i}\left(\left.\Pi\left(s^{1}, \ldots, s^{m}, r\right)\right|_{A}\right)=$ $\sum_{p_{k} \in A} \sum_{j=1}^{d_{k}} \alpha_{k j}^{i} \Pi_{k j}\left(s^{1}, \cdots s^{m}, r\right)$.

(iii) Security requirement: For any set $B \subset\left\{P_{1}, \cdots, P_{n}\right\}, T \subset\left\{S^{1}, \cdots, S^{m}\right\} \backslash$ $\left\{S^{i} \mid B \in A S_{i}, 1 \leq i \leq m\right\}$, it holds that $H(T \mid B)=H(T)$, where $H(\cdot)$ is the entropy function.

Similar to the equivalence relation of LSSS and MSP, Xiao and Liu [10] studied a corresponding relation between LMSSS and MSP computing multi-Boolean functions. Let $\mathcal{M}(\mathcal{K}, M, \psi)$ be a MSP with the $d \times l$ matrix $M$ and $f_{1}, \ldots, f_{m}$ : $\{0,1\}^{n} \rightarrow\{0,1\}$ be $m$ monotone Boolean functions. Suppose $\overrightarrow{v_{1}}, \ldots, \overrightarrow{v_{m}}$ are $m$ linear independent $l$-dimension vectors over $\mathcal{K}$, then it follows that $m \leq l$. In practice, we always have $m<l$ in order to use randombits. Then $\mathcal{M}$ can compute the Boolean functions $f_{1}, \ldots, f_{m}$ with respect to $\overrightarrow{v_{1}}, \ldots, \overrightarrow{v_{m}}$ if for any $1 \leq k \leq m$ and $1 \leq i_{1}<\cdots<i_{k} \leq m$, the following two conditions hold:

(i) For any $A \subseteq P, f_{i_{1}}\left(\overrightarrow{\delta_{A}}\right)=\cdots=f_{i_{k}}\left(\overrightarrow{\delta_{A}}\right)=1$ implies that $\overrightarrow{v_{j}} \in \operatorname{span}\left\{M_{A}\right\}$ for $1 \leq j \leq k$.

(ii) For any $A \subseteq P, f_{i_{1}}\left(\overrightarrow{\delta_{A}}\right)=\cdots=f_{i_{k}}\left(\overrightarrow{\delta_{A}}\right)=0$ implies that $\operatorname{Rank}\left(\begin{array}{c}M_{A} \\ \overrightarrow{v_{i_{1}}} \\ \vdots \\ \overrightarrow{v_{i_{k}}}\end{array}\right)=$ $\operatorname{Rank} M_{A}+k$. 
After a proper linear transform, any MSP computing the multi-Boolean function $f_{A S_{1}}, \cdots, f_{A S_{m}}$ with respect to $\overrightarrow{v_{1}}, \ldots, \overrightarrow{v_{m}}$ can be converted into a MSP computing the same multi-Boolean function with respect to $\overrightarrow{e_{1}}, \cdots, \overrightarrow{e_{m}}$, where $\overrightarrow{e_{i}}=(0, \ldots, 0, \stackrel{i}{1}, 0, \ldots, 0) \in \mathcal{K}^{l}$ for $1 \leq i \leq m$. So without loss of generality we always assume the target vectors are $\overrightarrow{e_{1}}, \cdots, \overrightarrow{e_{m}}$.

Theorem 1. [10] Let $A S_{1}, \cdots, A S_{m}$ be $m$ access structures over $P$ and $f_{A S_{1}}, \cdots$, $f_{A S_{m}}$ be the corresponding characteristic functions. Then there exists a linear multi-secret sharing scheme realizing $A S_{1}, \cdots, A S_{m}$ over a finite field $\mathcal{K}$ with size $d$ if and only if there exists a monotone span program computing monotone Boolean functions $f_{A S_{1}}, \cdots, f_{A S_{m}}$ with size $d$.

Actually, let $\mathcal{M}(\mathcal{K}, M, \psi)$ be a MSP computing monotone Boolean functions $f_{A S_{1}}, \cdots, f_{A S_{m}}$ with respect to $\overrightarrow{e_{1}}, \cdots, \overrightarrow{e_{m}}$, where $M$ is a $d \times l$ matrix. Then the corresponding LMSSS realizing $A S_{1}, \cdots, A S_{m}$ over $\mathcal{K}$ is as follows: For any multi-secret $\left(s^{1}, \ldots, s^{m}\right) \in \mathcal{K}^{m}$ and random input $\vec{\rho} \in \mathcal{K}^{l-m}$, the distribution function is defined by

$$
\Pi\left(s^{1}, \cdots, s^{m}, \vec{\rho}\right)=\left(\left(s^{1}, \cdots, s^{m}, \vec{\rho}\right)\left(M_{P_{1}}\right)^{\tau}, \cdots,\left(s^{1}, \cdots, s^{m}, \vec{\rho}\right)\left(M_{P_{n}}\right)^{\tau}\right),
$$

where " $\tau$ " denotes the transpose and $M_{P_{k}}$ denotes $M$ restricted to those rows $i$ with $\psi(i)=P_{k}, 1 \leq i \leq d, 1 \leq k \leq n$. As to reconstruction, since $\overrightarrow{e_{i}} \in$ $\operatorname{span}\left\{M_{A}\right\}$ for any $A \in A S_{i}$, i.e., there exists a vector $\vec{v}$ such that $\overrightarrow{e_{i}}=\vec{v} M_{A}$, then

$s^{i}=\left(s^{1}, \cdots, s^{m}, \vec{\rho}\right){\overrightarrow{e_{i}}}^{\tau}=\left(s^{1}, \cdots, s^{m}, \vec{\rho}\right)\left(\vec{v} M_{A}\right)^{\tau}=\left(s^{1}, \cdots, s^{m}, \vec{\rho}\right)\left(M_{A}\right)^{\tau} \vec{v}^{\tau}$,

where $\left(s^{1}, \cdots, s^{m}, \vec{\rho}\right)\left(M_{A}\right)^{\tau}$ are the shares held by players in $A$ and $\vec{v}$ can be computed by every participant.

\section{Parallel Multi-party Computation}

\subsection{Concepts and Notations}

The problem of secure MPC for one function has been studied by many people and it can be stated as follows: $n$ players $P_{1}, \ldots, P_{n}$ are to securely compute an agreed function $f\left(x_{1}, \ldots, x_{n}\right)=\left(y_{1}, \ldots, y_{n}\right)$ against an $\mathcal{A}$-adversary, where $P_{i}$ holds private input $x_{i}$ and is to get the output $y_{i}$. The security means that the correctness of the outputs and the privacy of players' inputs are always guaranteed no matter which set in $\mathcal{A}$ is corrupted by the adversary. In fact the function $f$ can be represented as $f=\left(f_{1}, \ldots, f_{n}\right)$ where $f_{i}\left(x_{1}, \ldots, x_{n}\right)=y_{i}$ for $1 \leq i \leq n$. As the general way of treating the MPC problem, we assume that the functions involved thereafter are all of the form of $f_{i}$. So the MPC problem can be seemed as securely computing $n$ functions with respect to the same adversary structure. As a natural extension, it is reasonable to consider securely computing multi-functions with respect to multi-adversary structures. Thus we propose the concept of secure parallel multi-party computation. 
Precisely, there are $m$ functions $f_{1}\left(x_{1}, \ldots, x_{n}\right), \ldots, f_{m}\left(x_{1}, \ldots, x_{n}\right)$ and $m$ corresponding adversary structures $\mathcal{A}_{1}, \ldots, \mathcal{A}_{m}$. For $1 \leq i \leq n$, player $P_{i}$ has private input $\left(x_{i}^{(1)}, x_{i}^{(2)}, \ldots, x_{i}^{(m)}\right)$, where $x_{i}^{(j)}$ is $P_{i}$ 's input to the function $f_{j}\left(x_{1}, \ldots, x_{n}\right)$. So the final value of $f_{j}$ is $f_{j}\left(x_{1}^{(j)}, x_{2}^{(j)}, \ldots, x_{n}^{(j)}\right)$. An $\left(\mathcal{A}_{1}, \ldots, \mathcal{A}_{m}\right)$-adversary can corrupt any set in $\mathcal{A}_{1} \cup \cdots \cup \mathcal{A}_{m}$. The $n$ players are to securely compute the multi-function $f_{1}, \ldots, f_{m}$ against an $\left(\mathcal{A}_{1}, \ldots, \mathcal{A}_{m}\right)$-adversary, that is, for any corrupted set $B \in \mathcal{A}_{i_{1}} \cap \cdots \cap \mathcal{A}_{i_{k}}$, where $1 \leq i_{1}<\cdots<i_{k} \leq m$ and $k \leq m$, functions $f_{i_{1}}, \ldots, f_{i_{k}}$ are securely computed, which includes the following two aspects:

(i) Correctness: For $1 \leq i \leq n, P_{i}$ finally gets the correct outputs of the functions $f_{i_{1}}, \ldots, f_{i_{k}}$.

(ii) Privacy: The adversary gets no information about other players' (players out of $B$ ) inputs for functions $f_{i_{1}}, \ldots, f_{i_{k}}$, except what can be implied from the inputs and outputs held by players in $B$.

The problem of secure parallel multi-party computation for the multi-function $f_{1}, \ldots, f_{m}$ against an $\left(\mathcal{A}_{1}, \ldots, \mathcal{A}_{m}\right)$-adversary is essentially a direct composition of problems of secure MPC for $f_{j}$ against an $\mathcal{A}_{j}$-adversary where $1 \leq j \leq m$. So it can be resolved by designing for each function and the corresponding adversary structure a secure MPC protocol and running them in a composite way. We call this a "direct sum" method. One of the results in 7] tells us that in the information theoretic model, every function can be securely computed against an adaptive, passive $\mathcal{A}$-adversary if and only if $\mathcal{A}$ is $\mathrm{Q} 2$, where $\mathrm{Q} 2$ is the condition that no two of the sets in the structure cover the full player set. Thus we evidently have the following proposition.

Proposition 1. In the information theoretic model, there exists a parallel multiparty computation protocol computing $m$ functions securely against an adaptive, passive $\left(\mathcal{A}_{1}, \ldots, \mathcal{A}_{m}\right)$-adversary if and only if $\mathcal{A}_{1}, \ldots, \mathcal{A}_{m}$ are all Q2.

Cramer et al. 3] build a secure MPC protocol for one function based on the multiplicative MSP computing one Boolean function. Here we extend it to the multiplicative MSP computing multi-Boolean functions. Precisely, let $\mathcal{M}(\mathcal{K}, M, \psi)$ be a MSP described in Section 2. Given two vectors $\vec{x}=\left(x_{1}, \ldots, x_{d}\right), \vec{y}=$ $\left(y_{1}, \ldots, y_{d}\right) \in \mathcal{K}^{d}$, we let $\vec{x} \diamond \vec{y}$ be the vector containing all entries of the form $x_{i} \cdot y_{j}$ with $\psi(i)=\psi(j)$, and $<\vec{x}, \vec{y}>$ denote the inner product. For example, let

$$
\vec{x}=\left(x_{11}, \ldots, x_{1 d_{1}}, \ldots, x_{n 1}, \ldots, x_{n d_{n}}\right), \quad \vec{y}=\left(y_{11}, \ldots, y_{1 d_{1}}, \ldots, y_{n 1}, \ldots, y_{n d_{n}}\right),
$$

where $\sum_{i=1}^{n} d_{i}=d$ and $x_{i 1}, \ldots, x_{i d_{i}}$, as well as $y_{i 1}, \ldots, y_{i d_{i}}$ are the entries distributed to $P_{i}$ according to $\psi$. Then $\vec{x} \diamond \vec{y}$ is the vector composed of the $\sum_{i=1}^{n} d_{i}^{2}$ entries $x_{i j} y_{i k}$, where $1 \leq j, k \leq d_{i}, 1 \leq i \leq n$, and $<\vec{x}, \vec{y}>=\sum_{i=1}^{n} \sum_{j=1}^{d_{i}} x_{i j} y_{i j}$. Using these notations, we give the following definition.

Definition 1. A monotone span program $\mathcal{M}(\mathcal{K}, M, \psi)$ computing Boolean functions $f_{1}, \ldots, f_{m}$ with respect to $\overrightarrow{e_{1}}, \cdots, \overrightarrow{e_{m}}$ is called multiplicative, if for $1 \leq i \leq$ $m$, there exists a $\sum_{i=1}^{n} d_{i}^{2}$-dimensional recombination vector $\overrightarrow{r_{i}}$, such that for any two multi-secrets $\left(s^{1}, \ldots, s^{m}\right),\left(s^{\prime 1}, \ldots, s^{\prime m}\right) \in \mathcal{K}^{m}$ and any $\vec{\rho}, \vec{\rho}^{\prime} \in \mathcal{K}^{l-m}$, it holds that 


$$
s^{i} s^{i}=<\overrightarrow{r_{i}},\left(s^{1}, \ldots, s^{m}, \vec{\rho}\right) M^{\tau} \diamond\left(s^{\prime 1}, \ldots, s^{\prime m}, \vec{\rho}^{\prime}\right) M^{\tau}>
$$

In fact, when $m=1$ the definition is the same as that of [3]. In the appendix we give an efficient and generic construction to build from any MSP a multiplicative MSP computing the same multi-Boolean function. Hence in the following we assume that the based MSP in Section 3.2 is already multiplicative.

\subsection{Construction from Any LMSSS}

In this section, assuming the adversary is passive and adaptive, we give a generic and efficient construction to obtain from any LMSSS a paralel multi-party computation protocol in the information theoretic model, provided that the access structures of the LMSSS allow MPC at all. Since LMSSS and MSP are equivalent, it turns out to be convenient to describe our protocol in terms of MSP's. We only describe the protocol in the case $m=2$ and it is a natural extension for $m>2$.

Suppose $\mathcal{A}_{1}$ and $\mathcal{A}_{2}$ are two adversary structures over $P$ and they are both Q2. For $1 \leq i \leq n$, player $P_{i}$ has private input $\left(x_{i}^{(1)}, x_{i}^{(2)}\right)$ and they are to jointly compute functions $f_{1}\left(x_{1}, \ldots, x_{n}\right)$ and $f_{2}\left(x_{1}, \ldots x_{n}\right)$. Let $A S_{1}=2^{P} \backslash \mathcal{A}_{1}, A S_{2}=$ $2^{P} \backslash \mathcal{A}_{2}$, and $\mathcal{M}(\mathcal{K}, M, \psi)$ be a multiplicative MSP computing Boolean functions $f_{A S_{1}}$ and $f_{A S_{2}}$ with respect to target vectors $\overrightarrow{e_{1}}, \overrightarrow{e_{2}}$, where $M$ is a $d \times l$ matrix over $\mathcal{K}$. How to construct such a MSP is out of concern in this paper. Next we describe our protocol in three phases: input sharing, computing and outputting.

INPUT SHARING. First each player shares his private input by using the MSP $\mathcal{M}(\mathcal{K}, M, \psi)$, i.e., for $1 \leq i \leq n$, player $P_{i}$ secretly and randomly selects $\overrightarrow{\rho_{i}}$ in the set of random inputs $R=\mathcal{K}^{l-2}$ and sends $\left(x_{i}^{(1)}, x_{i}^{(2)}, \overrightarrow{\rho_{i}}\right)\left(M_{P_{j}}\right)^{\tau}$ to player $P_{j}$, where $1 \leq j \leq n$ and $j \neq i$.

Computing. Since any function that is feasible to compute at all can be specified as a polynomial size arithmetic circuit over a finite field $\mathcal{K}$ with addition gates and multiplication gates, it is enough for us to discuss how to do additions and multiplications over $\mathcal{K}$. Different from computing a single function, in parallel multi-party computation, we compute the functions simultaneously other than one after another.

Precisely, suppose $f_{1}$ contains $p$ multiplications and $f_{2}$ contains $q$ multiplications, where $p \leq q$ and the multiplication considered here is operation between two elements. Then in each of the first $p$ steps, we compute two multiplications coming from the two functions, respectively. In each the following $q-p$ steps, we continue to compute a multiplications of $f_{2}$ and do nothing for $f_{1}$. So after $q$ steps we complete all the multiplications of both functions and get the intermediate results needed. Finally we compute all additions of both functions in one step. By doing so, we need less communication and random bits than the "direct sum" method. Furthermore, in order to guarantee security, all inputs and outputs of each step are multi-secret shared during computing and we call this condition the "invariant". 
Example 1. Let $P=\left\{P_{1}, P_{2}, P_{3}\right\}$, and $f_{1}=x_{2}^{2} x_{3}, f_{2}=x_{1} x_{2}+x_{3}$. For $1 \leq i \leq 3$, $P_{i}$ has private input $\left(x_{i}^{(1)}, x_{i}^{(2)}\right)$ which is multi-secret shared in the Input Sharing phase. Since $f_{1}$ contains two multiplications and $f_{2}$ contains one multiplication, the computing phase consists of three steps. The following table shows the computing process. Note that in the table, $x_{i}^{(j)}$ denotes an input value for the function $f_{j}$ held by $P_{i}, z_{i}^{(j)}$ denotes an intermediate value held by an imaginary player $I_{i}, x_{i}$ and $z_{i}$ are variables and $z_{i j}$ is the function to be computed at each step, where $1 \leq i \leq 3$ and $1 \leq j \leq 2$.

\begin{tabular}{l|c|c|c}
\hline & input & to compute & output \\
\hline & $\left(x_{1}^{(1)}, x_{1}^{(2)}\right)$ & & \\
Step 1 & $\left(x_{2}^{(1)}, x_{2}^{(2)}\right)$ \\
& $\left(x_{3}^{(1)}, x_{3}^{(2)}\right)$ & $\left(z_{11}=x_{2} x_{3}, z_{12}=x_{1} x_{2}\right)$ & $\left(z_{1}^{(1)}=x_{2}^{(1)} x_{3}^{(1)}, z_{1}^{(2)}=x_{1}^{(2)} x_{2}^{(2)}\right)$ \\
\hline \hline & $\left(x_{2}^{(1)}, x_{2}^{(2)}\right)$ & & $\left(z_{2}^{(1)}=x_{2}^{(1)} z_{1}^{(1)}, z_{2}^{(2)}=z_{1}^{(2)}\right)$ \\
Step 2 & $\left(z_{1}^{(1)}, z_{1}^{(2)}\right)$ & $\left(z_{21}=x_{2} z_{1}, z_{22}=z_{1}\right)$ & $\left(z_{3}^{(1)}, x_{3}^{(2)}\right)$ \\
\hline \hline & $\left(x_{3}^{(1)}, z_{2}^{(2)}\right)$ & $\left(z_{31}=z_{2}, z_{32}=z_{2}+x_{3}\right)$ & $\left(z_{3}^{(1)}=z_{2}^{(1)}, z_{3}^{(2)}=z_{2}^{(2)}+x_{3}^{(2)}\right)$ \\
\hline
\end{tabular}

In Step 1 we do two multiplications $x_{2} x_{3}$ and $x_{1} x_{2}$ for $f_{1}$ and $f_{2}$, respectively; in Step 2 we do a multiplication $x_{2} z_{1}$ for $f_{1}$ and do nothing for $f_{2}$; in Step 3 , we do an addition $z_{2}+x_{3}$ for $f_{2}$ and do nothing for $f_{1}$. It is evident that $z_{3}^{(1)}=x_{2}^{(1)} x_{2}^{(1)} x_{3}^{(1)}$ and $z_{3}^{(2)}=x_{1}^{(2)} x_{2}^{(2)}+x_{3}^{(2)}$. The invariant here means that for $1 \leq i \leq 3,\left(x_{i}^{(1)}, x_{i}^{(2)}\right),\left(z_{i}^{(1)}, z_{i}^{(2)}\right)$ all keep multi-secret shared by $\mathcal{M}(\mathcal{K}, M, \psi)$ during computing.

Next we discuss how to do multiplications or additions at each step. According to the type of operations we execute respectively for the two functions at each step (e.g. Step 1 of Example 11), there are four cases to be considered as follows, where "\" means that no operation is actually done and the output is one of the inputs. Without loss of generality, in the following we assume that $P=\left\{P_{1}, P_{2}, P_{3}, P_{4}\right\}$.

Case 1: $(+,+)$. First suppose that we are to compute $g_{1}=x_{1}+x_{2}$ and $g_{2}=x_{3}+x_{4}$. The inputs $\left(x_{i}^{(1)}, x_{i}^{(2)}\right)$ are multi-secret shared such that each player $P_{j}$ holds $\left(x_{i}^{(1)}, x_{i}^{(2)}, \overrightarrow{\rho_{i}}\right)\left(M_{P_{j}}\right)^{\tau}=\left(s_{i 1}^{(j)}, \ldots, s_{i d_{j}}^{(j)}\right) \in \mathcal{K}^{d_{j}}$ distributed by $P_{i}$ where $1 \leq i \leq 4$. The output is to be multi-secret shared $\left(x_{1}^{(1)}+x_{2}^{(1)}, x_{3}^{(2)}+x_{4}^{(2)}\right)$. Then $P_{j}$ locally computes:

$$
\begin{aligned}
& \left(x_{1}^{(1)}, x_{1}^{(2)}, \overrightarrow{\rho_{1}}\right)\left(M_{P_{j}}\right)^{\tau}+\left(x_{2}^{(1)}, x_{2}^{(2)}, \overrightarrow{\rho_{2}}\right)\left(M_{P_{j}}\right)^{\tau} \\
= & \left(x_{1}^{(1)}+x_{2}^{(1)}, x_{1}^{(2)}+x_{2}^{(2)}, \overrightarrow{\rho_{1}}+\overrightarrow{\rho_{2}}\right)\left(M_{P_{j}}\right)^{\tau} \\
= & \left(s_{11}^{(j)}+s_{21}^{(j)}, \ldots, s_{1 d_{j}}^{(j)}+s_{2 d_{j}}^{(j)}\right),
\end{aligned}
$$




$$
\begin{aligned}
& \left(x_{3}^{(1)}, x_{3}^{(2)}, \overrightarrow{\rho_{3}}\right)\left(M_{P_{j}}\right)^{\tau}+\left(x_{4}^{(1)}, x_{4}^{(2)}, \overrightarrow{\rho_{4}}\right)\left(M_{P_{j}}\right)^{\tau} \\
= & \left(x_{3}^{(1)}+x_{4}^{(1)}, x_{3}^{(2)}+x_{4}^{(2)}, \overrightarrow{\rho_{3}}+\overrightarrow{\rho_{4}}\right)\left(M_{P_{j}}\right)^{\tau} \\
= & \left(s_{31}^{(j)}+s_{41}^{(j)}, \ldots, s_{3 d_{j}}^{(j)}+s_{4 d_{j}}^{(j)}\right) .
\end{aligned}
$$

Actually, through (2) $P_{j}$ gets shares for $\left(x_{1}^{(1)}+x_{2}^{(1)}, x_{1}^{(2)}+x_{2}^{(2)}\right)$ and through (3) $P_{j}$ gets shares for $\left(x_{3}^{(1)}+x_{4}^{(1)}, x_{3}^{(2)}+x_{4}^{(2)}\right)$. In order to guarantee security, we need to multi-secret share $\left(x_{1}^{(1)}+x_{2}^{(1)}, x_{3}^{(2)}+x_{4}^{(2)}\right)$, each player must reshare his present shares. Precisely, by the reconstruction algorithm of the LMSSS, there exist $\vec{a}, \vec{b} \in \mathcal{K}^{\sum_{i=1}^{n} d_{i}}$, such that

$$
x_{1}^{(1)}+x_{2}^{(1)}=\sum_{j=1}^{n} \sum_{k=1}^{d_{j}} a_{j k}\left(s_{1 k}^{(j)}+s_{2 k}^{(j)}\right), \quad x_{3}^{(2)}+x_{4}^{(2)}=\sum_{j=1}^{n} \sum_{k=1}^{d_{j}} b_{j k}\left(s_{3 k}^{(j)}+s_{4 k}^{(j)}\right) .
$$

So each player $P_{j}$ reshares $\left(\sum_{k=1}^{d_{j}} a_{j k}\left(s_{1 k}^{(j)}+s_{2 k}^{(j)}\right), \sum_{k=1}^{d_{j}} b_{j k}\left(s_{3 k}^{(j)}+s_{4 k}^{(j)}\right)\right)$ through $\left(\sum_{k=1}^{d_{j}} a_{j k}\left(s_{1 k}^{(j)}+s_{2 k}^{(j)}\right), \sum_{k=1}^{d_{j}} b_{j k}\left(s_{3 k}^{(j)}+s_{4 k}^{(j)}\right), \vec{\rho}_{j}^{\prime}\right) M^{\tau}$ and sends each of other players a share. Finally $P_{j}$ adds up all his shares obtained from the resharing, i.e.,

$$
\begin{aligned}
& \sum_{i=1}^{n}\left(\sum_{k=1}^{d_{i}} a_{i k}\left(s_{1 k}^{(i)}+s_{2 k}^{(i)}\right), \sum_{k=1}^{d_{i}} b_{i k}\left(s_{3 k}^{(i)}+s_{4 k}^{(i)}\right),{\overrightarrow{\rho_{i}}}^{\prime}\right)\left(M_{P_{j}}\right)^{\tau} \\
= & \left(\sum_{i=1}^{n} \sum_{k=1}^{d_{i}} a_{i k}\left(s_{1 k}^{(i)}+s_{2 k}^{(i)}\right), \sum_{i=1}^{n} \sum_{k=1}^{d_{i}} b_{i k}\left(s_{3 k}^{(i)}+s_{4 k}^{(i)}\right), \sum_{i=1}^{n}{\overrightarrow{\rho_{i}}}^{\prime}\right)\left(M_{P_{j}}\right)^{\tau} \\
= & \left(x_{1}^{(1)}+x_{2}^{(1)}, x_{3}^{(2)}+x_{4}^{(2)}, \sum_{i=1}^{n} \vec{\rho}_{i}^{\prime}\right)\left(M_{P_{j}}\right)^{\tau},
\end{aligned}
$$

which is actually $P_{j}$ 's share for $\left(x_{1}^{(1)}+x_{2}^{(1)}, x_{3}^{(2)}+x_{4}^{(2)}\right)$.

Note that if we are to compute $\left(x_{1}^{(1)}+x_{2}^{(1)}, x_{1}^{(2)}+x_{2}^{(2)}\right)$ at this step, the equality (2) is enough and we do not need resharing any more. Although we only discuss adding up two items here, we can add up more items once in the same way. Furthermore, it is trivial to deal with multiplications with constants in $\mathcal{K}$, since the constant is public.

Case 2: $(\times, \times)$. Suppose we are to compute $\left(g_{1}=x_{1} x_{2}, g_{2}=x_{3} x_{4}\right)$. Since $\mathcal{M}(\mathcal{K}, M, \psi)$ is assumed to be multiplicative, there exist recombination vectors $\vec{r}, \vec{t} \in \mathcal{K}^{\sum_{i=1}^{n} d_{i}{ }^{2}}$, such that

$$
\begin{aligned}
& x_{1}^{(1)} x_{2}^{(1)}=<\vec{r},\left(x_{1}^{(1)}, x_{1}^{(2)}, \overrightarrow{\rho_{1}}\right) M^{\tau} \diamond\left(x_{2}^{(1)}, x_{2}^{(2)}, \overrightarrow{\rho_{2}}\right) M^{\tau}>, \\
& x_{3}^{(2)} x_{4}^{(2)}=<\vec{t},\left(x_{3}^{(1)}, x_{3}^{(2)}, \overrightarrow{\rho_{3}}\right) M^{\tau} \diamond\left(x_{4}^{(1)}, x_{4}^{(2)}, \overrightarrow{\rho_{4}}\right) M^{\tau}>.
\end{aligned}
$$

$P_{j}$ computes $\left(x_{1}^{(1)}, x_{1}^{(2)}, \overrightarrow{\rho_{1}}\right)\left(M_{P_{j}}\right)^{\tau} \diamond\left(x_{2}^{(1)}, x_{2}^{(2)}, \overrightarrow{\rho_{2}}\right)\left(M_{P_{j}}\right)^{\tau}=\left(\alpha_{j 1}, \ldots, \alpha_{j d_{j}^{2}}\right) \in$ $\mathcal{K}^{d_{j}^{2}}$ and $\left(x_{3}^{(1)}, x_{3}^{(2)}, \overrightarrow{\rho_{3}}\right)\left(M_{P_{j}}\right)^{\tau} \diamond\left(x_{4}^{(1)}, x_{4}^{(2)}, \overrightarrow{\rho_{4}}\right)\left(M_{P_{j}}\right)^{\tau}=\left(\beta_{j 1}, \ldots, \beta_{j d_{j}^{2}}\right) \in \mathcal{K}^{d_{j}^{2}}$. 
From (5) and (6) we have

$$
x_{1}^{(1)} x_{2}^{(1)}=\sum_{j=1}^{n} \sum_{k=1}^{d_{j}^{2}} r_{j k} \alpha_{j k}, \quad x_{3}^{(2)} x_{4}^{(2)}=\sum_{j=1}^{n} \sum_{k=1}^{d_{j}^{2}} t_{j k} \beta_{j k} .
$$

$P_{j}$ reshares $\left(\sum_{k=1}^{d_{j}^{2}} r_{j k} \alpha_{j k}, \sum_{k=1}^{d_{j}^{2}} t_{j k} \beta_{j k}\right)$ by $\left(\sum_{k=1}^{d_{j}^{2}} r_{j k} \alpha_{j k}, \sum_{k=1}^{d_{j}^{2}} t_{j k} \beta_{j k},{\overrightarrow{\rho_{j}}}^{\prime}\right) M^{\tau}$. Finally, $P_{j}$ computes

$$
\begin{aligned}
& \sum_{i=1}^{n}\left(\sum_{k=1}^{d_{i}^{2}} r_{i k} \alpha_{i k}, \sum_{k=1}^{d_{i}^{2}} t_{i k} \beta_{i k}, \vec{\rho}_{i}^{\prime}\right)\left(M_{P_{j}}\right)^{\tau} \\
= & \left(\sum_{i=1}^{n} \sum_{k=1}^{d_{i}^{2}} r_{i k} \alpha_{i k}, \sum_{i=1}^{n} \sum_{k=1}^{d_{i}{ }^{2}} t_{i k} \beta_{i k}, \sum_{i=1}^{n}{\overrightarrow{\rho_{i}}}^{\prime}\right)\left(M_{P_{j}}\right)^{\tau} \\
= & \left(x_{1}^{(1)} x_{2}^{(1)}, x_{3}^{(2)} x_{4}^{(2)}, \sum_{i=1}^{n} \vec{\rho}_{i}^{\prime}\right)\left(M_{P_{j}}\right)^{\tau},
\end{aligned}
$$

which is $P_{j}$ 's share for $\left(x_{1}^{(1)} x_{2}^{(1)}, x_{3}^{(2)} x_{4}^{(2)}\right)$.

Case 3: $(+, \backslash)$ or $(\backslash,+)$. Suppose we are to compute $\left(g_{1}=x_{1}+x_{2}, g_{2}=x_{3}\right)$. Similar to (4), we have $x_{3}^{(2)}=\sum_{j=1}^{n} \sum_{k=1}^{d_{j}} b_{j k} s_{3 k}^{(j)}$. So each player $P_{j}$ reshares $\left(\sum_{k=1}^{d_{j}} a_{j k}\left(s_{1 k}^{(j)}+s_{2 k}^{(j)}\right), \sum_{k=1}^{d_{j}} b_{j k} s_{3 k}^{(j)}\right)$ through

$$
\left(\sum_{k=1}^{d_{j}} a_{j k}\left(s_{1 k}^{(j)}+s_{2 k}^{(j)}\right), \sum_{k=1}^{d_{j}} b_{j k} s_{3 k}^{(j)}, \vec{\rho}_{j}^{\prime}\right) M^{\tau}
$$

and finally computes

$\sum_{i=1}^{n}\left(\sum_{k=1}^{d_{i}} a_{i k}\left(s_{1 k}^{(i)}+s_{2 k}^{(i)}\right), \sum_{k=1}^{d_{i}} b_{i k} s_{3 k}^{(i)}, \vec{\rho}_{i}^{\prime}\right)\left(M_{P_{j}}\right)^{\tau}=\left(x_{1}^{(1)}+x_{2}^{(1)}, x_{3}^{(2)}, \sum_{i=1} \vec{\rho}_{i}^{\prime}\right)\left(M_{P_{j}}\right)^{\tau}$, which is $P_{j}$ 's share for $\left(x_{1}^{(1)}+x_{2}^{(1)}, x_{3}^{(2)}\right)$.

Case 4: $(\times, \backslash)$ or $(\backslash, \times)$. It is similar to the above cases and details are omitted here.

Outputting. At the end of computing phase, we can see the final value $\left(f_{1}\left(x_{1}^{(1)}, \ldots, x_{n}^{(1)}\right), f_{2}\left(x_{1}^{(2)}, \ldots, x_{n}^{(2)}\right)\right)$ is multi-secret shared by using $\mathcal{M}$. If every player is allowed to get the value, in the last phase $P_{i}$ publics his share for $\left(f_{1}\left(x_{1}^{(1)}, \ldots, x_{n}^{(1)}\right), f_{2}\left(x_{1}^{(2)}, \ldots, x_{n}^{(2)}\right)\right)$ where $1 \leq i \leq n$, then every player can compute $\left(f_{1}\left(x_{1}^{(1)}, \ldots, x_{n}^{(1)}\right), f_{2}\left(x_{1}^{(2)}, \ldots, x_{n}^{(2)}\right)\right)$ by the reconstruction algorithm.

If $f_{1}\left(x_{1}^{(1)}, \ldots, x_{n}^{(1)}\right)$ is required to be held only by $P_{1}$ and $f_{2}\left(x_{1}^{(2)}, \ldots, x_{n}^{(2)}\right)$ is to be held only by $P_{2}$, all shares cannot be simply transmitted to $P_{1}$ and $P_{2}$. Because by doing so, $P_{1}$, resp. $P_{2}$ will also know $f_{2}\left(x_{1}^{(2)}, \ldots, x_{n}^{(2)}\right)$, resp. $f_{1}\left(x_{1}^{(1)}, \ldots, x_{n}^{(1)}\right)$. Fortunately, by the reconstruction algorithm, $f_{1}\left(x_{1}^{(1)}, \ldots, x_{n}^{(1)}\right)$ 
and $f_{2}\left(x_{1}^{(2)}, \ldots, x_{n}^{(2)}\right)$ are linear combinations of the shares that all players finally hold, so they can be computed through a simple MPC protocol 9] as follows, while keeping the privacy of the shares thus guaranteeing security for parallel MPC.

Since $\left(f_{1}\left(x_{1}^{(1)}, \ldots, x_{n}^{(1)}\right), f_{2}\left(x_{1}^{(2)}, \ldots, x_{n}^{(2)}\right)\right)$ is multi-secret shared through $\mathcal{M}$, suppose $P_{i}$ 's share for it is $\left(s_{i 1}, \cdots, s_{i d_{i}}\right) \in \mathcal{K}^{d_{i}}$ where $1 \leq i \leq n$. Similar to the equality (4), we have that

$$
f_{1}\left(x_{1}^{(1)}, \ldots, x_{n}^{(1)}\right)=\sum_{i=1}^{n} \sum_{k=1}^{d_{i}} a_{i k} s_{i k}, \quad f_{2}\left(x_{1}^{(2)}, \ldots, x_{n}^{(2)}\right)=\sum_{i=1}^{n} \sum_{k=1}^{d_{i}} b_{i k} s_{i k} .
$$

In order to securely compute $f_{1}\left(x_{1}^{(1)}, \ldots, x_{n}^{(1)}\right)$ such that only $P_{1}$ learns the value and other players get nothing new, we need a simple MPC protocol. Precisely, for $1 \leq i \leq n, P_{i}$ randomly selects $r_{i 1}, r_{i 2}, \cdots, r_{i(n-1)} \in \mathcal{K}$ and sets $r_{i n}=$ $\sum_{k=1}^{d_{i}} a_{i k} s_{i k}-\sum_{j=1}^{n-1} r_{i j}$. Then $P_{i}$ secretly transmits $r_{i j}$ to $P_{j}, 1 \leq j \leq n, j \neq i$. After that $P_{j}$ locally computes $\lambda_{j}=\sum_{i=1}^{n} r_{i j}$ and transmits $r_{j}$ to $P_{1}$ where $1 \leq j \leq n$. The process can be displayed as follows.

$$
\begin{array}{cccccc} 
& & P_{1} & \cdots & P_{n} \\
P_{1}: \sum_{k=1}^{d_{1}} a_{1 k} s_{1 k} & \rightarrow & r_{11} & \cdots & r_{1 n} & \sum_{k=1}^{d_{1}} a_{1 k} s_{1 k}=\sum_{j=1}^{n} r_{1 j} \\
P_{2}: \sum_{k=1}^{d_{2}} a_{2 k} s_{2 k} & \rightarrow & r_{21} & \cdots & r_{2 n} & \sum_{k=1}^{d_{2}} a_{2 k} s_{2 k}=\sum_{j=1}^{n} r_{2 j} \\
\cdots & \cdots & \cdots & \cdots & \cdots & \cdots \\
P_{n}: \sum_{k=1}^{d_{n}} a_{n k} s_{n k} & \rightarrow & r_{n 1} & \cdots & r_{n n} \quad \sum_{k=1}^{d_{n}} a_{n k} s_{n k}=\sum_{j=1}^{n} r_{n j} \\
& & \lambda_{1}=\sum_{i=1}^{n} r_{i 1} & \cdots & \lambda_{n}=\sum_{i=1}^{n} r_{i n}
\end{array}
$$

Finally, $P_{1}$ computes

$$
\sum_{j=1}^{n} \lambda_{j}=\sum_{j=1}^{n} \sum_{i=1}^{n} r_{i j}=\sum_{i=1}^{n} \sum_{j=1}^{n} r_{i j}=\sum_{i=1}^{n} \sum_{k=1}^{d_{i}} a_{i k} s_{i k}=f_{1}\left(x_{1}^{(1)}, \ldots, x_{n}^{(1)}\right) .
$$

Similarly, $f_{2}\left(x_{1}^{(2)}, \ldots, x_{n}^{(2)}\right)$ can be securely computed and only $P_{2}$ gets the final value.

\subsection{Comparing with the "Direct Sum" Method}

Since the "direct sum" method (in Section 3.1) is a natural way to realize secure parallel multi-party computation, we compare our protocol (in Section 3.2) with it. As to the security issue, note that in our protocol all inputs and outputs for every step is multi-secret shared during the protocol. For any $B \in \mathcal{A}_{i_{1}} \cap \cdots \cap \mathcal{A}_{i_{k}}$, it follows that $\left\{S^{i_{1}}, \ldots, S^{i_{k}}\right\} \subseteq\left\{S^{1}, \ldots, S^{m}\right\} \backslash\left\{S^{i} \mid B \in A S_{i}, 1 \leq i \leq m\right\}$. By the security requirement of the LMSSS, players in $B$ get no information about $\left\{S^{i_{1}}, \ldots, S^{i_{k}}\right\}$ from the shares they hold, that is, the intermediate communication data held by players in $B$ tells nothing about other players' inputs for functions $f_{i_{1}}, \ldots, f_{i_{k}}$. So an adversary corrupting players in $B$ gets 
no information about other players' (players out of $B$ ) inputs for functions $f_{i_{1}}, \ldots, f_{i_{k}}$, except what can be implied from the inputs and outputs held by players in $B$. Hence our protocol and the "direct sum" method are of the same security.

The communication complexity is an important criterion to evaluate a protocol. By using a " non-direct sum" LMSSS, our protocol may need less communication than the "direct sum" method, and this advantage becomes more evident when computing more complicated functions, i.e., the functions essentially contain more variables and more multiplications. In the next section, we show the advantage of communication complexity through a specific example.

\section{Example}

Suppose that $P=\left\{P_{1}, P_{2}, P_{3}, P_{4}, P_{5}\right\}$ is the set of players and $|\mathcal{K}|>5$. Let $A S_{1}=\left\{A \subset P|| A \mid \geq 2\right.$ and $\left.\left\{P_{1}, P_{2}\right\} \cap A \neq \varnothing\right\}$ and $A S_{2}=\{A \subset P|| A \mid \geq$ 2 and $\left.\left\{P_{4}, P_{5}\right\} \cap A \neq \varnothing\right\}$ be two access structures over $P$. The corresponding minimum access structures are as follows:

$$
\begin{aligned}
& \left(A S_{1}\right)_{m}=\left\{\left\{P_{1}, P_{2}\right\},\left\{P_{1}, P_{3}\right\},\left\{P_{1}, P_{4}\right\},\left\{P_{1}, P_{5}\right\},\left\{P_{2}, P_{3}\right\},\left\{P_{2}, P_{4}\right\},\left\{P_{2}, P_{5}\right\}\right\}, \\
& \left(A S_{2}\right)_{m}=\left\{\left\{P_{4}, P_{5}\right\},\left\{P_{1}, P_{4}\right\},\left\{P_{2}, P_{4}\right\},\left\{P_{3}, P_{4}\right\},\left\{P_{1}, P_{5}\right\},\left\{P_{2}, P_{5}\right\},\left\{P_{3}, P_{5}\right\}\right\} .
\end{aligned}
$$

Obviously, the two corresponding adversary structures $\mathcal{A}_{1}=2^{P} \backslash A S_{1}$ and $\mathcal{A}_{2}=$ $2^{P} \backslash A S_{2}$ are both Q2. The players are to securely compute multi-functions $f_{1}=x_{1}+x_{2} x_{3}, f_{2}=x_{1} x_{2}$ against an $\left(\mathcal{A}_{1}, \mathcal{A}_{2}\right)$-adversary. For $1 \leq i \leq 5$, player $P_{i}$ has private input $\left(x_{i}^{(1)}, x_{i}^{(2)}\right)$.

By the "direct sum" method, we need to design for $f_{i}$ a MPC protocol against an $\mathcal{A}_{i}$-adversary where $1 \leq i \leq 2$. From 3 we know that the key step is to devise LSSS with respect to $A S_{1}$ and $A S_{2}$, respectively. let

$$
M_{1}=\left(\begin{array}{ll}
1 & 1 \\
2 & 1 \\
0 & 1 \\
0 & 1 \\
0 & 1
\end{array}\right), \quad M_{2}=\left(\begin{array}{ll}
0 & 1 \\
0 & 1 \\
0 & 1 \\
1 & 1 \\
2 & 1
\end{array}\right),
$$

and $\psi_{1}, \psi_{2}:\{1,2, \cdots, 5\} \rightarrow P$ be defined as $\psi_{1}(i)=\psi_{2}(i)=P_{i}$ for $1 \leq i \leq 5$. It is easy to verify that $\mathcal{M}_{i}\left(\mathcal{K}, M_{i}, \psi_{i}\right)$ is a multiplicative MSP computing $f_{A S_{i}}$ with respect to $(1,0) \in \mathcal{K}^{2}$ where $1 \leq i \leq 2$. Then the MPC protocol follows. Note that the MPC protocol for computing a single function also has input sharing phase, computing phase and outputting phase. 
By the protocol in Sec3.2, first we need to design a LMSSS with respect to the multi-access structure $A S_{1}, A S_{2}$. Let $M=\left(\begin{array}{cccc}1 & 0 & 1 & 1 \\ 0 & 0 & 0 & 1 \\ 0 & 0 & 0 & 1 \\ 2 & 0 & 1 & 1 \\ 0 & 0 & 1 & 1 \\ 0 & 0 & 1 & 0 \\ 0 & 1 & -2 & -1 \\ 0 & 0 & 2 & 1 \\ 0 & 1 & -1 & -1\end{array}\right)$ and $\psi:\{1,2, \ldots, 9\} \rightarrow$ $P$ be defined as $\psi(1)=\psi(2)=P_{1}, \psi(3)=\psi(4)=P_{2}, \psi(5)=P_{3}, \psi(6)=$ $\psi(7)=P_{4}, \psi(8)=\psi(9)=P_{5}$. It can be verified that $\mathcal{M}(\mathcal{K}, M, \psi)$ is a MSP computing $f_{A S_{1}}$ and $f_{A S_{2}}$ with respect to the target vectors $\overrightarrow{e_{1}}, \overrightarrow{e_{2}}$, and later we are to verify that $\mathcal{M}(\mathcal{K}, M, \psi)$ is multiplicative.

InPut Sharing. First for $1 \leq i \leq 3, P_{i}$ multi-secret share his private input $\left(x_{i}^{(1)}, x_{i}^{(2)}\right)$ by randomly choosing $\alpha_{i}, \beta_{i} \in \mathcal{K}$ and sending $\left(x_{i}^{(1)}, x_{i}^{(2)}, \alpha_{i}, \beta_{i}\right)\left(M_{P_{j}}\right)^{\tau}$ to player $P_{j}$, where $1 \leq j \leq n$. The following table shows the shares each player holds for $\left(x_{i}^{(1)}, x_{i}^{(2)}\right)$ after the phase.

\begin{tabular}{c|c|c|c}
\hline & $\left(x_{1}^{(1)}, x_{1}^{(2)}\right)$ & $\left(x_{2}^{(1)}, x_{2}^{(2)}\right)$ & $\left(x_{3}^{(1)}, x_{3}^{(2)}\right)$ \\
\hline$P_{1}$ & $x_{1}^{(1)}+\alpha_{1}+\beta_{1}, \beta_{1}$ & $x_{2}^{(1)}+\alpha_{2}+\beta_{2}, \beta_{2}$ & $x_{3}^{(1)}+\alpha_{3}+\beta_{3}, \beta_{3}$ \\
\hline$P_{2}$ & $\beta_{1}, 2 x_{1}^{(1)}+\alpha_{1}+\beta_{1}$ & $\beta_{2}, 2 x_{2}^{(1)}+\alpha_{2}+\beta_{2}$ & $\beta_{3}, 2 x_{3}^{(1)}+\alpha_{3}+\beta_{3}$ \\
\hline$P_{3}$ & $\alpha_{1}+\beta_{1}$ & $\alpha_{2}+\beta_{2}$ & $\alpha_{3}+\beta_{3}$ \\
\hline$P_{4}$ & $\alpha_{1}, x_{1}^{(2)}-2 \alpha_{1}-\beta_{1}$ & $\alpha_{2}, x_{2}^{(2)}-2 \alpha_{2}-\beta_{2}$ & $\alpha_{3}, x_{3}^{(2)}-2 \alpha_{3}-\beta_{3}$ \\
\hline$P_{5}$ & $2 \alpha_{1}+\beta_{1}, x_{1}^{(2)}-\alpha_{1}-\beta_{1}$ & $2 \alpha_{2}+\beta_{2}, x_{2}^{(2)}-\alpha_{2}-\beta_{2}$ & $2 \alpha_{3}+\beta_{3}, x_{3}^{(2)}-\alpha_{3}-\beta_{3}$ \\
\hline
\end{tabular}

Denote $\left(x_{i}^{(1)}, x_{i}^{(2)}, \alpha_{i}, \beta_{i}\right) M^{\tau}=\left(s_{i 1}^{(1)}, s_{i 2}^{(1)}, s_{i 1}^{(2)}, s_{i 2}^{(2)}, s_{i 1}^{(3)}, s_{i 1}^{(4)}, s_{i 2}^{(4)}, s_{i 1}^{(5)}, s_{i 2}^{(5)}\right)$, that is, $P_{j}$ holds $s_{i k}^{(j)}$ for $\left(x_{i}^{(1)}, x_{i}^{(2)}\right)$ where $1 \leq k \leq d_{i}, 1 \leq j \leq 5$.

It can be verified that

$$
\begin{aligned}
\begin{aligned}
& x_{1}^{(1)}=\left(x_{1}^{(1)}+\alpha_{1}+\beta_{1}\right)-\left(\alpha_{1}+\beta_{1}\right), \quad x_{1}^{(2)}=\left(\alpha_{1}+\beta_{1}\right)+\alpha_{1}+\left(x_{1}^{(2)}-2 \alpha_{1}-\beta_{1}\right) . \\
& x_{2}^{(1)} x_{3}^{(1)}=-\left(x_{2}^{(1)}+\alpha_{2}+\beta_{2}\right)\left(x_{3}^{(1)}+\alpha_{3}+\beta_{3}\right)+ \\
& \frac{1}{2}\left(2 x_{2}^{(1)}+\alpha_{2}+\beta_{2}\right)\left(2 x_{3}^{(1)}+\alpha_{3}+\beta_{3}\right)+\frac{1}{2}\left(\alpha_{2}+\beta_{2}\right)\left(\alpha_{3}+\beta_{3}\right),(10) \\
& x_{1}^{(2)} x_{2}^{(2)}=\left(\alpha_{1}+\beta_{1}\right)\left(\alpha_{2}+\beta_{2}\right)-\alpha_{1} \alpha_{2}+\left(x_{1}^{(2)}-2 \alpha_{1}-\beta_{1}\right)\left(x_{2}^{(2)}-2 \alpha_{2}-\beta_{2}\right)+ \\
&\left(2 \alpha_{1}+\beta_{1}\right)\left(x_{2}^{(2)}-\alpha_{2}-\beta_{2}\right)+\left(x_{1}^{(2)}-\alpha_{1}-\beta_{1}\right)\left(2 \alpha_{2}+\beta_{2}\right) .
\end{aligned}
\end{aligned}
$$

The equality (9) gives the reconstruction algorithms for $\left\{P_{1}, P_{3}\right\}$ to recover $x_{1}^{(1)}$ and for $\left\{P_{3}, P_{4}\right\}$ to recover $x_{1}^{(2)}$, so as in the equality (4), we can set

$$
\vec{a}=(1,0,0,0,-1,0,0,0,0), \vec{b}=(0,0,0,0,1,1,1,0,0) .
$$


The equalities (10) and (11) show the MSP $\mathcal{M}(\mathcal{K}, M, \psi)$ is multiplicative. Precisely, if we have

$$
\begin{aligned}
& \left(x_{1}^{(1)}, x_{1}^{(2)}, \alpha_{1}, \beta_{1}\right) M^{\tau} \diamond\left(x_{2}^{(1)}, x_{2}^{(2)}, \alpha_{2}, \beta_{2}\right) M^{\tau} \\
= & \left(s_{11}^{(1)} s_{21}^{(1)}, s_{11}^{(1)} s_{22}^{(1)}, s_{12}^{(1)} s_{21}^{(1)}, s_{12}^{(1)} s_{22}^{(1)}, s_{11}^{(2)} s_{21}^{(2)}, s_{11}^{(2)} s_{22}^{(2)}, s_{12}^{(2)} s_{21}^{(2)}, s_{12}^{(2)} s_{22}^{(2)}, s_{11}^{(3)} s_{21}^{(3)},\right. \\
& \left.s_{11}^{(4)} s_{21}^{(4)}, s_{11}^{(4)} s_{22}^{(4)}, s_{12}^{(4)} s_{21}^{(4)}, s_{12}^{(4)} s_{22}^{(4)}, s_{11}^{(5)} s_{21}^{(5)}, s_{11}^{(5)} s_{22}^{(5)}, s_{12}^{(5)} s_{21}^{(5)}, s_{12}^{(5)} s_{22}^{(5)}\right),
\end{aligned}
$$

then as in the equality (17) the recombination vectors are as follows:

$$
\begin{aligned}
\vec{r} & =\left(-1,0,0,0,0,0,0, \frac{1}{2}, \frac{1}{2}, 0,0,0,0,0,0,0,0\right), \\
\vec{t} & =(0,0,0,0,0,0,0,0,1,-1,0,0,1,0,1,1,0) .
\end{aligned}
$$

We transmit $22 \log |\mathcal{K}|$ bits of information in this phase. For simplicity, the functions computed in this example involve a few variables. If all variables are involved in each function, i.e., variables $x_{1}, \ldots, x_{5}$ all appear in each function, then we need to transmit $36 \log |\mathcal{K}|$ bits in the input sharing phase, while by the "direct sum" method $40 \log |\mathcal{K}|$ bits need to be transmitted in this phase.

Computing. This phase consists of two steps.

Step 1: $(x, x)$. The output of this step is to be the multi-secret shared $\left(x_{2}^{(1)} x_{3}^{(1)}, x_{1}^{(2)} x_{2}^{(2)}\right)$. From (10) and (11), we can see that in the recombination vector $\vec{r}$ only $P_{1}, P_{2}$ and $P_{3}$ has nonzero coefficients, and in the recombination vector $\vec{t}$ only $P_{3}, P_{4}$ and $P_{5}$ has nonzero coefficients, so $P_{1}$ reshares $\left(u_{1}, v_{1}\right)=\left(-\left(x_{2}^{(1)}+\alpha_{2}+\beta_{2}\right)\left(x_{3}^{(1)}+\alpha_{3}+\beta_{3}\right), 0\right), P_{2}$ reshares $\left(u_{2}, v_{2}\right)=\left(\frac{1}{2}\left(2 x_{2}^{(1)}+\right.\right.$ $\left.\left.\alpha_{2}+\beta_{2}\right)\left(2 x_{3}^{(1)}+\alpha_{3}+\beta_{3}\right), 0\right), P_{3}$ reshares $\left(u_{3}, v_{3}\right)=\left(\frac{1}{2}\left(\alpha_{2}+\beta_{2}\right)\left(\alpha_{3}+\beta_{3}\right),\left(\alpha_{1}+\right.\right.$ $\left.\left.\beta_{1}\right)\left(\alpha_{2}+\beta_{2}\right)\right), P_{4}$ reshares $\left(u_{4}, v_{4}\right)=\left(0,-\alpha_{1} \alpha_{2}+\left(x_{1}^{(2)}-2 \alpha_{1}-\beta_{1}\right)\left(x_{2}^{(2)}-2 \alpha_{2}-\beta_{2}\right)\right)$ and $P_{5}$ reshares $\left(u_{5}, v_{5}\right)=\left(0,\left(2 \alpha_{1}+\beta_{1}\right)\left(x_{2}^{(2)}-\alpha_{2}-\beta_{2}\right)+\left(x_{1}^{(2)}-\alpha_{1}-\beta_{1}\right)\left(2 \alpha_{2}+\beta_{2}\right)\right)$.

After resharing, as shares of $\left(u_{i}, v_{i}\right), P_{1}$ gets $u_{i}+\alpha_{i}^{\prime}+\beta_{i}^{\prime}, \beta_{i}^{\prime} ; P_{2}$ gets $\beta_{i}^{\prime}, 2 u_{i}+$ $\alpha_{i}^{\prime}+\beta_{i}^{\prime} ; P_{3}$ gets $\alpha_{i}^{\prime}+\beta_{i}^{\prime} ; P_{4}$ gets $\alpha_{i}^{\prime}, v_{i}-2 \alpha_{i}^{\prime}-\beta_{i}^{\prime}$ and $P_{5}$ gets $2 \alpha_{i}^{\prime}+\beta_{i}^{\prime}, v_{i}-\alpha_{i}^{\prime}-\beta_{i}^{\prime}$, where $1 \leq i \leq 5$. Finally

$P_{1}$ computes $\sum_{i=1}^{5}\left(u_{i}+\alpha_{i}^{\prime}+\beta_{i}^{\prime}\right)=x_{2}^{(1)} x_{3}^{(1)}+\sum_{i=1}^{5}\left(\alpha_{i}^{\prime}+\beta_{i}^{\prime}\right), \quad$ and $\sum_{i=1}^{5} \beta_{i}^{\prime}$; $P_{2}$ computes $\sum_{i=1}^{5} \beta_{i}^{\prime}$, and $\sum_{i=1}^{5}\left(2 u_{i}+\alpha_{i}^{\prime}+\beta_{i}^{\prime}\right)=2 x_{2}^{(1)} x_{3}^{(1)}+\sum_{i=1}^{5}\left(\alpha_{i}^{\prime}+\beta_{i}^{\prime}\right)$; $P_{3}$ computes $\sum_{i=1}^{5}\left(\alpha_{i}^{\prime}+\beta_{i}^{\prime}\right)$;

$P_{4}$ computes $\sum_{i=1}^{5} \alpha_{i}^{\prime}$, and $\sum_{i=1}^{5}\left(v_{i}-2 \alpha_{i}^{\prime}-\beta_{i}^{\prime}\right)=x_{1}^{(2)} x_{2}^{(2)}-\sum_{i=1}^{5}\left(2 \alpha_{i}^{\prime}+\beta_{i}^{\prime}\right)$; $\left.\beta_{i}^{\prime}\right)$.

$P_{5}$ computes $\sum_{i=1}^{5}\left(2 \alpha_{i}^{\prime}+\beta_{i}^{\prime}\right)$, and $\sum_{i=1}^{5}\left(v_{i}-\alpha_{i}^{\prime}-\beta_{i}^{\prime}\right)=x_{1}^{(2)} x_{2}^{(2)}-\sum_{i=1}^{5}\left(\alpha_{i}^{\prime}+\right.$ It can be verified that they are the shares for $\left(x_{2}^{(1)} x_{3}^{(1)}, x_{1}^{(2)} x_{2}^{(2)}\right)$ generated from $M\left(x_{2}^{(1)} x_{3}^{(1)}, x_{1}^{(2)} x_{2}^{(2)}, \sum_{i=1}^{5} \alpha_{i}^{\prime}, \sum_{i=1}^{5} \beta_{i}^{\prime}\right)^{\tau}$.

Step 2: $(+, \backslash)$. The output of this step is to be multi-secret shared $\left(x_{1}^{(1)}+\right.$ $\left.x_{2}^{(1)} x_{3}^{(1)}, x_{1}^{(2)} x_{2}^{(2)}\right)$. Since $\left(x_{2}^{(1)} x_{3}^{(1)}, x_{1}^{(2)} x_{2}^{(2)}\right)$ is multi-secret shared after Step 1 and $\left(x_{1}^{(1)}, x_{1}^{(2)}\right)$ is multi-secret shared in the Input Sharing phase, then each player adds his shares for $\left(x_{2}^{(1)} x_{3}^{(1)}, x_{1}^{(2)} x_{2}^{(2)}\right)$ to his shares for $\left(x_{1}^{(1)}, x_{1}^{(2)}\right)$. By the linear 
combinations given in (9), $P_{1}$ reshares $\left(p_{1}, q_{1}\right)=\left(\left(x_{1}^{(1)}+\alpha_{1}+\beta_{1}\right)+x_{2}^{(1)} x_{3}^{(1)}+\right.$ $\left.\sum_{i=1}^{5}\left(\alpha_{i}^{\prime}+\beta_{i}^{\prime}\right), 0\right), P_{3}$ reshares $\left(p_{3}, q_{3}\right)=\left(-\left(\alpha_{1}+\beta_{1}\right)-\sum_{i=1}^{5}\left(\alpha_{i}^{\prime}+\beta_{i}^{\prime}\right), \sum_{i=1}^{5}\left(\alpha_{i}^{\prime}+\right.\right.$ $\left.\left.\beta_{i}^{\prime}\right)\right)$ and $P_{4}$ reshares $\left(p_{4}, q_{4}\right)=\left(0, \sum_{i=1}^{5} \alpha_{i}^{\prime}+x_{1}^{(2)} x_{2}^{(2)}-\sum_{i=1}^{5}\left(2 \alpha_{i}^{\prime}+\beta_{i}^{\prime}\right)\right)$. Finally, $P_{1}$ computes $\sum_{i=1,3,4}\left(p_{i}+\alpha_{i}^{\prime \prime}+\beta_{i}^{\prime \prime}\right)=x_{1}^{(1)}+x_{2}^{(1)} x_{3}^{(1)}+\sum_{i=1,3,4}\left(\alpha_{i}^{\prime \prime}+\beta_{i}^{\prime \prime}\right), \quad$ and $\sum_{i=1,3,4} \beta_{i}^{\prime \prime}$

$P_{2}$ computes $\sum_{i=1,3,4} \beta_{i}^{\prime \prime}$, and $\sum_{i=1,3,4}\left(2 p_{i}+\alpha_{i}^{\prime \prime}+\beta_{i}^{\prime \prime}\right)=2\left(x_{1}^{(1)}+x_{2}^{(1)} x_{3}^{(1)}\right)+$ $\sum_{i=1,3,4}\left(\alpha_{i}^{\prime \prime}+\beta_{i}^{\prime \prime}\right)$

$P_{3}$ computes $\sum_{i=1,3,4}\left(\alpha_{i}^{\prime \prime}+\beta_{i}^{\prime \prime}\right)$

$P_{4}$ computes $\sum_{i=1,3,4} \alpha_{i}^{\prime \prime}$, and $\sum_{i=1,3,4}\left(q_{i}-2 \alpha_{i}^{\prime \prime}-\beta_{i}^{\prime \prime}\right)=x_{1}^{(2)} x_{2}^{(2)}-\sum_{i=1,3,4}\left(2 \alpha_{i}^{\prime \prime}+\beta_{i}^{\prime \prime}\right)$; $P_{5}$ computes $\sum_{i=1,3,4}\left(2 \alpha_{i}^{\prime \prime}+\beta_{i}^{\prime \prime}\right)$, and $\sum_{i=1,3,4}\left(q_{i}-\alpha_{i}^{\prime \prime}-\beta_{i}^{\prime \prime}\right)=x_{1}^{(2)} x_{2}^{(2)}-$ $\sum_{i=1,3,4}\left(\alpha_{i}^{\prime \prime}+\beta_{i}^{\prime \prime}\right)$

It can be verified that they are the shares for $\left(x_{1}^{(1)}+x_{2}^{(1)} x_{3}^{(1)}, x_{1}^{(2)} x_{2}^{(2)}\right)$ generated from $M\left(x_{1}^{(1)}+x_{2}^{(1)} x_{3}^{(1)}, x_{1}^{(2)} x_{2}^{(2)}, \sum_{i=1,3,4} \alpha_{i}^{\prime \prime}, \sum_{i=1,3,4} \beta_{i}^{\prime \prime}\right)^{\tau}$.

In each step dealing with multiplications, our protocol transmits at most $36 \log |\mathcal{K}|$ bits of information. By the "direct sum" method, each time we do a multiplication it need to transmit $28 \log |\mathcal{K}|$ bits. Assume that $f_{1}$ contains $p$ multiplications and $f_{2}$ contains $q$ multiplications, where $p \leq q$. Then our protocol need transmit $36 q \log |\mathcal{K}|$ bits to complete all multiplications, while the "direct sum" method transmits $20(p+q) \log |\mathcal{K}|$ bits. If $p=q$, we see that our protocol transmits $4 p \log |\mathcal{K}|$ bits less than the "direct sum" method.

In the last step of this phase, that is, when we do additions, from the reconstruction algorithm given by (9) only $P_{1}, P_{3}$ and $P_{4}$ need to reshare their shares. But by the "direct sum" method, no resharing is needed when doing additions. So our protocol transmits at most $22 \log |\mathcal{K}|$ bits more than the "direct sum" method when dealing with additions. However, when both functions essentially contain large numbers of multiplications, our protocol has great advantage in communication complexity.

Outputting. Assume that all players are allowed to get the final value of both functions. Then every player publics his share for $\left(x_{1}^{(1)}+x_{2}^{(1)} x_{3}^{(1)}, x_{1}^{(2)} x_{2}^{(2)}\right)$ and can compute the final value by the reconstruction algorithms. If $x_{1}^{(1)}+x_{2}^{(1)} x_{3}^{(1)}$ is assumed to be held by $P_{1}$ and $x_{1}^{(2)} x_{2}^{(2)}$ is assumed to be held by $P_{2}$, then our protocol transmits at most $20 \log |\mathcal{K}|$ bits more than the "direct sum" method according to (8). Fortunately, this disadvantage is fixed, that is, it does not depend on the functions we compute. 
As a whole, our protocol needs less communication than the "direct sum" method when computing complicated functions.

\section{References}

1. Beimel, A.: Secure Schemes for Secret Sharing and Key Distribution. PhD thesis, Technion - Israel Institute of Techonlogy, 1996. Available on Internet http://www.cs.bgu.ac.il/ beimel/pub.html

2. Blundo, C., De Santis, A., Di Crescenzo, G.,: Multi-Secret sharing schemes. Advances in Crypotology-CRYPTO'94. LNCS, Vol. 839, 150-163, 1995.

3. Cramer, R., Damgard, I., Maurer, U.: General Secure Multi-Party Computation from any Linear Secret-Sharing Scheme. Proc. EUROCRYPT '00, Springer Verlag LNCS, vol. 1807, pp. 316-334. Full version available from IACR eprint archive, 2000.

4. Ding, C., Laihonen, T., Renvall, A.,: Linear multi-secret sharing schemes and errorcorrecting codes. Journal of Universal Computer Science, Vol.3, No.9, 1997, 10231036

5. Fehr, S.,: Efficient Construction of Dual MSP. manuscript 1999. Available on Internet http://homepages.cwi.nl/ fehr/papers/Feh99.pdf

6. Goldreich, O., Micali, S., Wigderson, A.: How to play ANY mental game.Proceedings of the nineteenth annual ACM conference on Theory of computing, pp.218-229, January 1987, New York, New York, United States.

7. Hirt, M., Maurer, U.,: Player simulation and general adversary structures in perfect multi-party computation. Journal of Cryptology, vol.13, NO. 1, pp.31-60, 2000.

8. Karchmer, M. and Wigderson, A.: On span programs. Proc. 8th Ann. Symp. Structure in complexity Theory, IEEE 1993, pp. 102-111.

9. Xiao, L.,: Secret sharing schemes: theory and application. PhD thesis, Academy of Mathematics and Systems Science, CAS, 2004.

10. Xiao, L., Liu, M.: Linear Multi-secret sharing schemes. Science in China Ser. F Information Sciences, vol. 48, NO.1, pp. 125-136, 2005.

11. Yao, A.: Protocols for Secure Computation. Proc. of IEEE FOGS '82, pp. 160-164, 1982 .

\section{Appendix: Construct Multiplicative MSP}

Let $\mathcal{M}(\mathcal{K}, M, \psi)$ be a MSP computing $f_{A S_{1}}$ and $f_{A S_{2}}$ with respect to $\left\{\overrightarrow{e_{1}}, \overrightarrow{e_{2}}\right\}$. For simplicity, we use $\overrightarrow{e_{1}}$, resp. $\overrightarrow{e_{2}}$, to denote vectors with the form $(1,0, \cdots, 0)$, resp. $(0,1,0, \cdots, 0)$, without distinguishing the dimensions, and the dimension can be determined from context. From 5 we can assume that the columns of $M$ are linear independent and so $d \geq l$. Compute $\overrightarrow{w_{1}}, \overrightarrow{w_{2}}$ be such that $\overrightarrow{w_{1}} M=\overrightarrow{e_{1}}$ and $\overrightarrow{w_{2}} M=\overrightarrow{e_{2}}$, and compute $\overrightarrow{v_{1}}, \ldots, \overrightarrow{v_{d-l}}$ as a basis of the solution space to the linear functions $\vec{v} M=\overrightarrow{0}$. Then construct a matrix

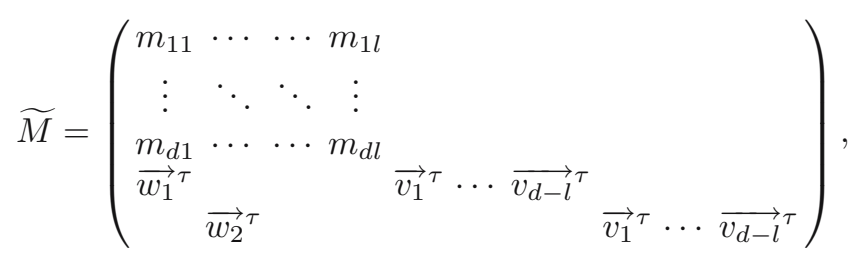


where $\left(\begin{array}{ccc}m_{11} & \cdots & m_{1 l} \\ \vdots & \ddots & \vdots \\ m_{d 1} & \cdots & m_{d l}\end{array}\right)=M$, and the blanks in $\widetilde{M}$ denote zero elements. So $\widetilde{M}$ is a $3 d \times(2 d-l)$ matrix over $\mathcal{K}$. Define a function $\widetilde{\psi}:\{1, \ldots, 3 d\} \rightarrow\{1, \ldots, n\}$ as follows: For $1 \leq k \leq d, \widetilde{\psi}(k)=\psi(k)$; For $d<k \leq 2 d$, $\widetilde{\psi}(k)=\psi(k-d)$; For $2 d<k \leq 3 d, \widetilde{\psi}(k)=\psi(k-2 d)$. Therefore we get a $\operatorname{MSP} \widetilde{\mathcal{M}}(\mathcal{K}, \widetilde{M}, \widetilde{\psi})$.

Proposition 2. The monotone span program $\widetilde{\mathcal{M}}(\mathcal{K}, \widetilde{M}, \widetilde{\psi})$ constructed above is a multiplicative MSP computing Boolean functions $f_{A S_{1}}$ and $f_{A S_{2}}$ with respect to target vectors $\left\{\overrightarrow{e_{1}}, \overrightarrow{e_{2}}\right\}$.

Proof: Let $M_{1}^{*}$, resp. $M_{2}^{*}$ be the matrix composed of rows from the $(d+1)$ th to the $2 d$ th row of $\widetilde{M}$, resp. from the $(2 d+1)$ th to the $3 d$ th row of $\widetilde{M}$. Then $M_{1}^{*}$ and $M_{2}^{*}$ are two $d \times(2 d-l)$ matrices, and $\widetilde{M}=\left(\begin{array}{c}M 0 \\ M_{1}^{*} \\ M_{2}^{*}\end{array}\right)$, where $M 0$ denotes the $d \times(2 d-l)$ matrix generated by adding $2(d-l)$ all zero columns to the right of the original $d \times l$ matrix $M$. Let $A S_{1}^{*}=\left\{B \subset P \mid \bar{B} \notin A S_{1}\right\}$ and $A S_{2}^{*}=\left\{B \subset P \mid \bar{B} \notin A S_{2}\right\}$. From [5], the $M S P \mathcal{M}_{1}^{*}\left(\mathcal{K}, M_{1}^{*}, \psi\right)$, resp. $\mathcal{M}_{2}^{*}\left(\mathcal{K}, M_{2}^{*}, \psi\right)$ computes the Boolean function $f_{A S_{1}^{*}}$, resp. $f_{A S_{2}^{*}}$ with respect to the target vector $\overrightarrow{e_{1}}$, resp. $\overrightarrow{e_{2}}$.

In order to prove that $\widetilde{\mathcal{M}}(\mathcal{K}, \widetilde{M}, \widetilde{\psi})$ computes Boolean functions $f_{A S_{1}}$ and $f_{A S_{2}}$ with respect to target vectors $\left\{\overrightarrow{e_{1}}, \overrightarrow{e_{2}}\right\}$, we need to prove: (1) $\overrightarrow{e_{1}} \in \operatorname{span}\left\{\widetilde{M}_{A}\right\}$ iff $A \in A S_{1}$; (2) $\overrightarrow{e_{2}} \in \operatorname{span}\left\{\widetilde{M}_{A}\right\}$ iff $A \in A S_{2}$; (3) If $A \notin A S_{1} \cup A S_{2}$, then $\widetilde{M}$ rejects $A$ with respect to $\left\{\overrightarrow{e_{1}}, \overrightarrow{e_{2}}\right\}$, ie. Rank $\left(\begin{array}{c}\vec{M}_{A} \\ \overrightarrow{e_{1}} \\ \overrightarrow{e_{2}}\end{array}\right)=\operatorname{Rank} \widetilde{M}_{A}+2$.

(1) Suppose that $A \in A S_{1}$. Because $\mathcal{M}(\mathcal{K}, M, \psi)$ computes $f_{A S_{1}}$ with respect to $\overrightarrow{e_{1}}, \overrightarrow{e_{1}} \in \operatorname{span}\left\{(M 0)_{A}\right\} \subset \operatorname{span}\left\{\widetilde{M}_{A}\right\}$. On the other hand, suppose that $\overrightarrow{e_{1}} \in$ $\operatorname{span}\left\{\widetilde{M}_{A}\right\}$. If $\overrightarrow{e_{1}} \in \operatorname{span}\left\{(M 0)_{A}\right\}$, then $A \in A S_{1}$ because $\mathcal{M}$ computes $f_{A S_{1}}$ with respect to $\overrightarrow{e_{1}}$. Otherwise $\left(M_{1}^{*}\right)_{A}$ or $\left(M_{2}^{*}\right)_{A}$ must contribute to the generation of $\overrightarrow{e_{1}}$. If $\left(M_{1}^{*}\right)_{A}$ contributes, it is easy to see that its contribution must be $\operatorname{span}\left\{\overrightarrow{e_{1}}\right\}$. So $\overrightarrow{e_{1}} \in \operatorname{span}\left\{\left(M_{1}^{*}\right)_{A}\right\}$. Because $\mathcal{M}_{1}^{*}\left(\mathcal{K}, M_{1}^{*}, \psi\right)$ computes the Boolean function $f_{A S_{1}^{*}}$ with respect to the target vector $\overrightarrow{e_{1}}, \overrightarrow{e_{1}} \in \operatorname{span}\left\{\left(M_{1}^{*}\right)_{A}\right\}$ implies that $A \in$ $A S_{1}^{*}$. By the assumption $\mathcal{A}_{1}=2^{P}-A S_{1}$ is $Q 2, A S_{1}^{*} \subset A S_{1}$ and then $A \in A S_{1}$. Similarly, if $\left(M_{2}^{*}\right)_{A}$ contributes, its contribution must be $\operatorname{span}\left\{\overrightarrow{e_{2}}\right\}$. So $\overrightarrow{e_{2}} \in$ $\operatorname{span}\left\{\left(M_{2}^{*}\right)_{A}\right\}$, and thus $A \in A S_{2}$. Because $\mathcal{M}(\mathcal{K}, M, \psi)$ computes $f_{A S_{2}}$ with respect to $\overrightarrow{e_{2}}$, then $\overrightarrow{e_{2}} \in \operatorname{span}\left\{M_{A}\right\}$. As a result, the contribution of $\left(M_{2}^{*}\right)_{A}$ is included in that of $(M 0)_{A}$. Thus we can disregard $\left(M_{2}^{*}\right)_{A}$ when generating $\overrightarrow{e_{1}}$, and we have proved that $\overrightarrow{e_{1}} \in \operatorname{span}\left\{(M 0)_{A},\left(M_{2}^{*}\right)_{A}\right\}$ implies $A \in A S_{1}$.

(2)By the discussion similar to (1), $\overrightarrow{e_{2}} \in \operatorname{span}\left\{\widetilde{M}_{A}\right\}$ iff $A \in A S_{2}$;

(3) Suppose that $A \notin A S_{1} \cup A S_{2}$. It follows that

$$
\begin{aligned}
& \operatorname{span}\left\{(M 0)_{A}, \overrightarrow{e_{1}}, \overrightarrow{e_{2}}\right\} \cap \operatorname{span}\left\{\left(M_{1}^{*}\right)_{A}\right\}=\operatorname{span}\left\{(M 0)_{A}, \overrightarrow{e_{1}}, \overrightarrow{e_{2}}\right\} \cap \operatorname{span}\left\{\left(M_{2}^{*}\right)_{A}\right\} \\
&=\operatorname{span}\left\{\left(M_{1}^{*}\right)_{A}\right\} \cap \operatorname{span}\left\{\left(M_{2}^{*}\right)_{A}\right\}=0 .
\end{aligned}
$$


So

$$
\begin{aligned}
\operatorname{Rank}\left(\begin{array}{c}
\widetilde{M}_{A} \\
\overrightarrow{e_{1}} \\
\overrightarrow{e_{2}}
\end{array}\right) & =\operatorname{Rank}\left(\begin{array}{c}
(M 0)_{A} \\
\overrightarrow{e_{1}} \\
\overrightarrow{e_{2}}
\end{array}\right)+\operatorname{Rank}\left(M_{1}^{*}\right)_{A}+\operatorname{Rank}\left(M_{2}^{*}\right)_{A} \\
& =\operatorname{Rank}(M 0)_{A}+2+\operatorname{Rank}\left(M_{1}^{*}\right)_{A}+\operatorname{Rank}\left(M_{2}^{*}\right)_{A} \\
& =\operatorname{Rank} \widetilde{M}_{A}+2,
\end{aligned}
$$

where the equality (13) and (15) come from the equality (12), and the equality (14) comes from the fact that $\mathcal{M}$ computes $f_{A S_{1}}$ and $f_{A S_{2}}$ with respect to $\left\{\overrightarrow{e_{1}}, \overrightarrow{e_{2}}\right\}$.

Then we prove that $\widetilde{\mathcal{M}}(\mathcal{K}, \widetilde{M}, \widetilde{\psi})$ is multiplicative. For any $s_{1}, s_{1}^{\prime} \in S^{1}, s_{2}, s_{2}^{\prime} \in$ $S^{2}$, and $\vec{\rho}, \vec{\rho}^{\prime} \in \mathcal{K}^{2 d-l-2}$, denote

$$
\left(s_{1}, s_{2}, \vec{\rho}\right) \widetilde{M}^{\tau}=\left(s_{1}, s_{2}, \vec{\rho}\right)\left((M 0)^{\tau},\left(M_{1}^{*}\right)^{\tau},\left(M_{2}^{*}\right)^{\tau}\right)=(\vec{u}, \vec{v}, \vec{w}),
$$

where $\vec{u}=\left(s_{1}, s_{2}, \vec{\rho}\right)(M 0)^{\tau} \in \mathcal{K}^{d}, \vec{v}=\left(s_{1}, s_{2}, \vec{\rho}\right)\left(M_{1}^{*}\right)^{\tau} \in \mathcal{K}^{d}$ and $\vec{w}=$ $\left(s_{1}, s_{2}, \vec{\rho}\right)\left(M_{2}^{*}\right)^{\tau} \in \mathcal{K}^{d}$. Then using the operation notations in Section 3.1, we have the following:

$$
\begin{aligned}
& <\vec{u}, \vec{v}^{\prime}>=\vec{u} \vec{v}^{\prime \tau}=\left(s_{1}, s_{2}, \vec{\rho}\right) M^{\tau} M_{1}^{*}\left(\begin{array}{c}
s_{1}^{\prime} \\
s_{2}^{\prime} \\
\vec{\rho}^{\prime \tau}
\end{array}\right) \\
& =\left(s_{1}, s_{2}, \vec{\rho}\right)\left(\begin{array}{cccc}
1 & 0 & \cdots & 0 \\
0 & 0 & \cdots & 0 \\
\vdots & \vdots & \ddots & \vdots \\
0 & 0 & \cdots & 0
\end{array}\right)\left(\begin{array}{c}
s_{1}^{\prime} \\
s_{2}^{\prime} \\
\vec{\rho}^{\prime \tau}
\end{array}\right)=s_{1} s_{1}^{\prime} \text {, } \\
& <\vec{u}, \vec{w}^{\prime}>=\vec{u} \vec{w}^{\prime \tau}=\left(s_{1}, s_{2}, \vec{\rho}\right) M^{\tau} M_{2}^{*}\left(\begin{array}{c}
s_{1}^{\prime} \\
s_{2}^{\prime} \\
\vec{\rho}^{\prime \prime}
\end{array}\right) \\
& =\left(s_{1}, s_{2}, \vec{\rho}\right)\left(\begin{array}{ccccc}
0 & 0 & 0 & \cdots & 0 \\
0 & 1 & 0 & \cdots & 0 \\
0 & 0 & 0 & \cdots & 0 \\
\vdots & \vdots & \vdots & \ddots & \vdots \\
0 & 0 & 0 & \cdots & 0
\end{array}\right)\left(\begin{array}{c}
s_{1}^{\prime} \\
s_{2}^{\prime} \\
\vec{\rho}^{\prime \tau}
\end{array}\right)=s_{2} s_{2}^{\prime} .
\end{aligned}
$$

Hence $\widetilde{\mathcal{M}}(\mathcal{K}, \widetilde{M}, \widetilde{\psi})$ is multiplicative. 